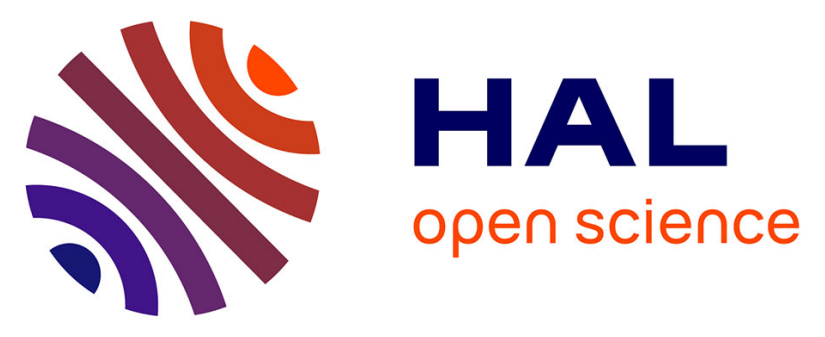

\title{
Carrier scattering processes and low energy phonon spectroscopy in hybrid perovskites crystals
}

Jacky Even, Serge Paofai, Philippe Bourges, Antoine Létoublon, Stéphane Cordier, Olivier Durand, Claudine Katan

\section{- To cite this version:}

Jacky Even, Serge Paofai, Philippe Bourges, Antoine Létoublon, Stéphane Cordier, et al.. Carrier scattering processes and low energy phonon spectroscopy in hybrid perovskites crystals. Proceedings of SPIE, the International Society for Optical Engineering, 2016, Physics, Simulation, and Photonic Engineering of Photovoltaic Devices V, 9743, pp.97430M. 10.1117/12.2213623 . hal-01291680

\section{HAL Id: hal-01291680 \\ https://hal-univ-rennes1.archives-ouvertes.fr/hal-01291680}

Submitted on 21 Mar 2016

HAL is a multi-disciplinary open access archive for the deposit and dissemination of scientific research documents, whether they are published or not. The documents may come from teaching and research institutions in France or abroad, or from public or private research centers.
L'archive ouverte pluridisciplinaire HAL, est destinée au dépôt et à la diffusion de documents scientifiques de niveau recherche, publiés ou non, émanant des établissements d'enseignement et de recherche français ou étrangers, des laboratoires publics ou privés.

$$
\text { Copyright }
$$




\title{
Carrier scattering processes and low energy phonon spectroscopy in hybrid perovskites crystals
}

\author{
Jacky Even*a , Serge Paofai ${ }^{\mathrm{b}}$, Philippe Bourges ${ }^{\mathrm{c}}$, Antoine Letoublon ${ }^{\mathrm{a}}$, Stéphane Cordier ${ }^{\mathrm{b}}$, Olivier \\ Durand $^{\mathrm{a}}$, Claudine Katan ${ }^{\mathrm{b}}$ \\ ${ }^{a}$ Université Européenne de Bretagne, INSA, FOTON UMR 6082, 35708 Rennes, France; ${ }^{b}$ Institut \\ des Sciences Chimiques de Rennes, UMR 6226, CNRS - Université de Rennes 1, France; \\ ${ }^{c}$ Laboratoire Léon Brillouin, UMR12 CEA-CNRS, Bât. 563 CEA Saclay, 91191 Gif sur Yvette \\ Cedex, France
}

\begin{abstract}
Despite the wealth of research conducted the last three years on hybrid organic perovskites (HOP), several questions remain open including: to what extend the organic moiety changes the properties of the material as compared to allinorganic (AIP) related perovskite structures. To ultimately reach an answer to this question, we have recently introduced two approaches that were designed to take the stochastic molecular degrees of freedom into account, and suggested that the high temperature cubic phase of HOP and AIP is an appropriate reference phase to rationalize HOP's properties. In this paper, we recall the main concepts and discuss more specifically the various possible couplings between charge carriers and low energy excitations such as acoustic and optical phonons. As available experimental or simulated data on low energy excitations are limited, we also present preliminary neutron scattering and ultrasonic measurements obtained and freshly prepared single crystals of $\mathrm{CH}_{3} \mathrm{NH}_{3} \mathrm{PbBr}_{3}$.
\end{abstract}

Keywords: hybrid perovskite, electron-phonon interaction, transport, neutron scattering, ultrasonic measurements

\section{INTRODUCTION}

Following the recent breakthrough of hybrid organic perovskites (HOP) for low-cost photovoltaic (PV) devices, there has been a wealth of fundamental and applied research devoted to this class of materials[1][2][3][4][5][6][7][8][9][10][11]. Nonetheless, there are still many open fundamental open questions related to issue relevant to devices under working conditions. Among those, it is yet unclear whether or not "hybrid" is required to actually reach appropriate optoelectronic properties suited for PV technologies. Early studies on methylammonium (MA) lead-halide perovskites have clearly evidence the tumbling between various orientations of the organic cation, with a typical timescale of picoseconds [12][13][14][15]. This dynamics has been predicted to induce a dramatic reduction of the exciton binding energy due to dielectric screening [16], which has been confirmed experimentally [17][18]. To further rationalize effects of both the stochastic molecular orientational dynamics and the frozen static configurations on material properties, symmetry-based discrete pseudo-spin (PS) and continuous rotator functions have recently been introduced [19][20]. This has permitted the establishment of the criteria necessary for the coupling between molecular degrees of freedom and order parameters relevant to ferroelectric or antiferro-distorsive phase transitions. It also provides a framework to explore the linear coupling between acoustic or optical phonons to stochastic molecular degrees of freedom [20][21]. Meanwhile, little is known about vibrational modes in HOP, especially in the low energy part of the spectrum. For instance, there have been a few experimental and theoretical papers on Raman spectroscopy of HOP but with possible decomposition of the samples due to the significant photo-sensitivity of the material and the low frequency modes have only been addressed very recently but still above $50 \mathrm{~cm}^{-1}[22]$. Conversely, phonon dispersion curves have already been investigated along time ago for related all-inorganic halide perovskites (AIP), especially in $\mathrm{CsPbCl}_{3}$ by means of coherent neutron scattering measurements[24]. In the cubic phase, they reveal a very low energy $(<2 \mathrm{meV})$ for the whole acoustic phonon density of states, and damped optical phonons undergoing an overdamping near the $X, M$ and $R$ points of the Brillouin zone (BZ), which can be explained by a strong anharmonicity induced by the light halogen atoms[23]. Unfortunately, a similar detailed study on the phonon dynamics of 3D HOP is still lacking, but comparable strongly anisotropic thermal ellipsoids for halides have already been reported[25][26][26].Incoherent neutron scattering experiments have been performed recently, and the measured

*jacky.even@insa-rennes.fr

citation: Jacky Even ; Serge Paofai ; Philippe Bourges ; Antoine Létoublon ; Stéphane Cordier, Olivier Durand, Claudine Katan, " Carrier scattering processes and low energy phonon spectroscopy in hybrid perovskites crystals ", Proc. SPIE 9743, Physics, Simulation, and Photonic Engineering of Photovoltaic Devices V, 97430M (March 14, 2016); doi:10.1117/12.2213623; http://dx.doi.org/10.1117/12.2213623 
quasielastic scattering yielded information about molecular relaxational modes at high temperature in $\mathrm{CH}_{3} \mathrm{NH}_{3} \mathrm{PbI}_{3}$ and $\mathrm{CH}_{3} \mathrm{NH}_{3} \mathrm{PbBr}_{3}$ powders[27][28][29].

In this paper, we will first outline the approaches developed to tackle the stochastic molecular degrees of freedom. On one hand, the concept of PS offers a discrete symmetry-adapted representation of molecular cation's orientations that is able to account for both elastic and electric multipoles[19][20]. On the other hand, rotator functions provide symmetryadapted continuous variables that are also well suited to account for the tumbling motion of the molecular axis in HOP, in the same way as they had for the $\mathrm{CN}^{-}$anion in $\mathrm{NaCN}$ [20][21]. From this and the Fermi golden combined to symmetry considerations allows to draw a general classification of the possible electronic couplings for AIP and HOP in the cubic reference phase. Next, we present preliminary coherent neutron scattering and ultrasonic measurements, which constitute the first report of data related to acoustic modes in HOP. These data have been recorded on a freshly synthetized $\mathrm{CH}_{3} \mathrm{NH}_{3} \mathrm{PbBr}_{3}$ single crystals. Crystal growth has been performed according to the inverse temperature crystallization recipe developed by M. I. Saidaminov et al.[30], except for the heating procedure using an oven instead of an oil bath.

\section{THEORETICAL ANALYSIS OF CARRIER COUPLING TO LOW ENERGY EXCITATIONS}

We first introduce a symmetry-based analysis of the Pm-3m high temperature cubic reference phase that incorporates both the intrinsic disorder and translational symmetry of the lattice[16][19][20]. It relies on a description of the electronic states that includes (i) effect of spin-orbit coupling (SOC), (ii) acoustic and optical phonons (Figure 1a) and (iii) stochastic molecular reorientations (Figure 1b), assuming a separation of the molecular translational (center of mass) and rotational degrees of freedom. Noteworthy, as a first approximation it neglects the linear rotational-vibrational coupling in the lattice, which is allowed by symmetry in the cubic phase. Such linear coupling can be accounted for in a second step in the modelling of the dynamical properties, since translational and orientational excitations may have the same irreducible representations (IR)[19][20].
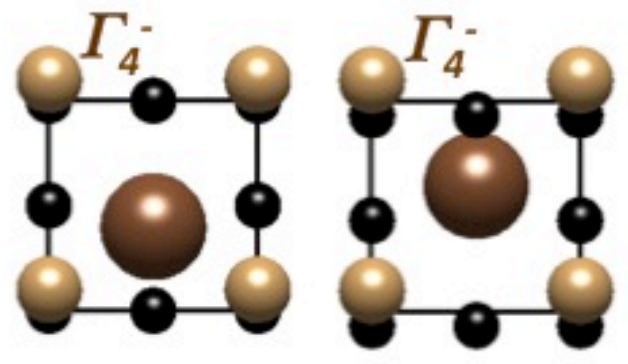

(a)

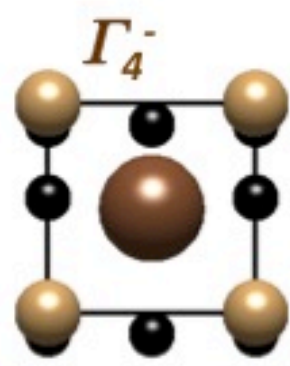

(b)

b)

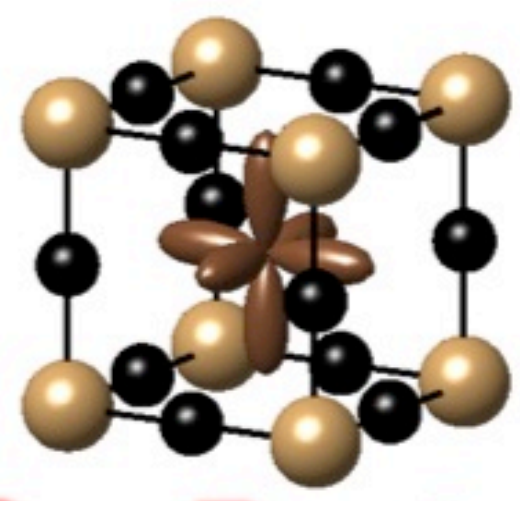

Figure 1: (a) Schematic representation of atomic translations for one of the triply degenerated $\Gamma_{4}^{-}$polar optical mode of the Pm-3m hybrid perovskite cubic phase. The average shape of the molecules at the center of the unit cell is represented by large dark brown spheres, halogen atoms along the edges correspond to the small black spheres and metal atoms in the corners are shown as medium spheres. (b) Schematic representation of a molecular pseudospin (PS) at the center of the cubic cell $\left(\Gamma_{I}^{+}\right.$irreducible representation), when the maximum of the orientational probability distribution of the molecular dipole is oriented towards the cubic cell facets.

To take into account for the stochastic orientational molecular degrees of freedom, with long- or short-range correlations, continuous rotator functions and a simplified discrete pseudospin (PS) representation of the cations are an attractive solution[19][20][21]. In a local cubic environment, the discrete sets of symmetry equivalent molecular orientations can be classified into six scenarios. The simplest first three correspond to molecular dipoles pointing towards cubic cell facets (Figure 1b), a halogen atom or the center of an octahedron. In the PS picture a discrete variable is defined for each possible molecular orientation (all orientations defining the multiplicity of the variable/considered scenario), and amounts to 1 or 0 depending if the specific orientation is occupied or not. Symmetry-adapted 
combinations of these discrete variables can then be constructed for a single crystal cell, which can be decomposed along the irreducible representations of the simple (without SOC) or double (with SOC) point group[9][16][19]. For instance, at the $\Gamma$ point, the PS IR decomposition reads $\Gamma_{1}^{+}+\Gamma_{3}^{+}+\Gamma_{4}^{-}$when molecules are preferentially pointing toward cubic cell facets. The symmetric (+) and asymmetric (-) PS IR correspond to elastic and electric multipoles, respectively. Elastic quadrupoles and electric dipoles are represented by the IR $\Gamma_{3}^{+}$and $\Gamma_{4}^{-}$, respectively. Representative cases of cations in AIP and HOP are $\mathrm{Cs}^{+}, \mathrm{FA}^{+}$(formamidinium) and $\mathrm{MA}^{+}$(methylammonium). They correspond respectively to the case where one has a sole electric monopoles, additional elastic multipoles, and both elastic and electric multipoles. The elastic multipole reflects the shape of the molecular cation (dumbbell-like for MA), mainly related to steric interactions, whereas the electric dipole may be strong (MA, 2.3D), much smaller (FA, 0.2D) or even vanishing for specific cations such as GA (guanidinium). The positive charge (electric monopole) carried by the molecular center of mass preserves $\mathrm{Pm}-3 \mathrm{~m}$ symmetry, e.g. $\mathrm{Cs}^{+}$in AIP. We stress that for both electric and elastic effects, spherical symmetry is not mandatory and corresponds to an oversimplification. Unlike monopoles, molecular elastic and electric multipoles do not necessarily preserve Pm-3m symmetry and entail implementing a suitable approach, which is afforded by the PS framework.

A more complete description of the symmetry properties of the molecular excitations at low energy is possible thanks to stochastic rotator functions[20][21]. Molecular rotator functions are symmetry adapted functions able to change continuously when the orientations is changing. For instance, a combination of spherical harmonics will be suited to describe a linear molecule, which is a good approximation for the $\mathrm{CN}$ axis of MA. The rotation of methyl or ammonium groups can be further included by means of Wigner rotations matrices. Rotator functions allow deriving probability distributions that can be used to rationalize data obtained by diffraction experiments or simulations. As an example, dipolar electric and quadrupolar elastic autocorrelation functions computed from molecular dynamics trajectories helped to confront the DFT simulations and incoherent neutron scattering studies of $\mathrm{MAPbX}_{3}$ compounds [20].

In the context of photovoltaic issues, it is of tremendous importance to determine the possible coupling between photoexcited species, both excitons and free charge carriers, and atomic collective modes (phonons) and/or molecular degrees of freedom. Using the Fermi golden-rule, the symmetry analysis of reveals that both elastic PS and polar PS mechanism are symmetry allowed (Table 1). The symmetry analysis of electron-phonon scattering processes reveals that polar phonons (Fröhlich FOP), as well as deformation potentials due to acoustic phonons (ADP) are of major concern for charge carrier mobilities in HOP (Table 1). Meanwhile, polar acoustic phonon mechanisms related to piezoelectricity (PZA) and non-polar optical phonon (ODP) mechanisms related to deformation potentials are predicted to vanish in the cubic Pm-3m reference phase. This result out of symmetry considerations shows that AIP and HOP form a new class of semiconductors with specific properties. By comparison, the piezoelectric electron-phonon (PZA) coupling is important in III-V semiconductors both for conduction band minimum (CBM) and valence band maximum (VBM) states, while it vanishes in group IV semiconductors for symmetry reasons. The Fröhlich interaction between delocalized CBM and VBM states and polar optical phonons (FOP) is especially important in AIP and HOP, because these materials are highly ionic by comparison to conventional III-V semiconductors (GaAs, InP, ...).

citation: Jacky Even; Serge Paofai ; Philippe Bourges ; Antoine Létoublon ; Stéphane Cordier, Olivier Durand, Claudine Katan, " Carrier scattering processes and low energy phonon spectroscopy in hybrid perovskites crystals ", Proc. SPIE 9743, Physics, Simulation, and Photonic Engineering of Photovoltaic Devices V, 97430M (March 14, 2016); doi:10.1117/12.2213623; http://dx.doi.org/10.1117/12.2213623 


\begin{tabular}{|c|c|c|}
\hline $\begin{array}{c}\text { Low energy lattice excitations } \\
\text { (Pm3m cubic phase) }\end{array}$ & $\begin{array}{c}\text { AIP }\left(\mathrm{CsPbX}_{3}\right) \\
\mathrm{CBM} \text { and VBM } \\
\text { (SOC included })\end{array}$ & $\begin{array}{c}\text { HOP }\left(\mathrm{MAPbX}_{3} \text { and FAPbX }\right. \\
\text { CBM and VBM } \\
\text { (SOC included) }\end{array}$ \\
\hline Acoustic phonons & ADP & ADP \\
\hline Optical Phonons & FOP & FPS \\
\hline Molecular Elastic Pseudospins & - & PPS \\
\hline Molecular Polar Pseudospins & - & \\
\hline
\end{tabular}

Table 1: General classification of the possible electronic couplings for all-inorganic (AIP) and hybrid (HOP) perovskites in the cubic Pm3m reference phase. Possible couplings of conduction band minimum (CBM) and valence band maximum (VBM) band edge electronic states including spin-orbit coupling (SOC) with non-polar acoustic phonons

(ADP), polar optical phonons (FOP) or elastic (EPS) and polar (PPS) pseudospins at $\Gamma$ point. Polar piezo-acoustic phonon (PZA) and non-polar optical phonon (ODP) processes are predicted to vanish by symmetry.

The Fermi golden rule can be used to computed numerically the scattering processes, and the charge mobilities are deduced from approximate equations for parabolic bands. The previously computed wavefunctions at the CBM and VBM were used for that purpose. FOP is the dominant mechanism in $\mathrm{MAPbI}_{3}$. It leads to RT carrier mobilities on the order of 80 and $120 \mathrm{~cm}^{2} \mathrm{~V}^{-1} \mathrm{~s}^{-1}$ for electrons and holes respectively (figure 2), in reasonable agreement with the highest experimental values reported so far.

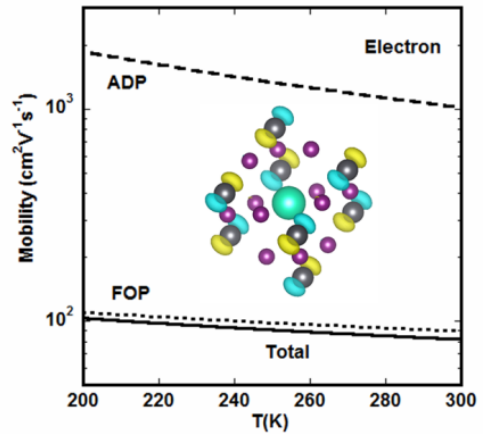

(a)

Figure 2: Temperature dependence of electron (a) and hole (b) mobilities in $\mathrm{MAPbI}_{3}$. The acoustic phonon (ADP process, dashed lines), polar optical phonon (FOP process, dotted lines), contributions to the total mobilities (solid lines) are indicated. The previously computed [9][16] wavefunctions at the conduction band minimum (CBM) and valence band maximum VBM used to compute the Fermi-golden rule scattering elements are given in insert (here the spin-orbit coupling (SOC) is not accounted for to simplify the representations). 


\section{NEUTRON SCATTERING STUDY OF MAPbBr 3}

Coherent inelastic neutron scattering experiment has been perfomed using the triple-axis spectrometer 4F1 located on a cold-neutron source at the reactor Orphée (Laboratoire Léon Brillouin, CEA-Saclay) [31]. The incident and scattered beams were focused by a pyrolytic graphite 002 double-monochromator and analyzer, respectively. The collimation conditions were open. The final neutron wave vector was held fixed at $k_{f}=1.55 \mathrm{~A}^{-1}$. A beryllium filter cooled with liquid nitrogen was placed after the sample to remove high-order neutrons from the beam.

To measure phonons, it is necessary to grow large single crystals. Various single crystals of $\mathrm{MAPbBr}_{3}$ have been synthetized, purified and grown according to Ref. [30]. A large-size $\left(\sim 1 \mathrm{~cm}^{3}\right)$ single crystal $\mathrm{MAPbBr}_{3}$ has been used for the inelastic neutron scattering. The sample being protonated, a large background due to the incoherent neutron cross section of hydrogen was observed. The sample was mounted on a close-cycle refrigerator installed on the spectrometer sample table, ensuring a temperature stability less than $0.1 \mathrm{~K}$.

The sample was mounted in a scattering plane such that reciprocal directions $(1,0,0)$ and $(0,1,1)$ were within the horizontal plane. We quote the wave-vector $\mathbf{Q}=(\mathrm{H}, \mathrm{K}, \mathrm{L})$ in units of cubic lattice vector $\mathrm{a}^{*}=2 \pi / \mathrm{a} \sim 1.06 \mathrm{~A}^{-1}(\mathrm{a}=5.91 \AA$ is the lattice parameter). Bragg reflections of the room temperature cubic phase $Q=(H, K, K)$ are then accessible (figure 3a). This has been chosen in order to reach the superlattice reflections appearing in both the tetragonal and orthorhombic low temperature phases, which are occurring respectively at the $M(1 / 2,1 / 2,0)$ and $R(1 / 2,1 / 2,1 / 2)$ or equivalents points. The cubic to tetragonal phase transition occurs first around $\mathrm{T}_{\mathrm{c}} \sim 225 \mathrm{~K}$ in $\mathrm{MAPbBr}_{3}[32]$.

We perform the intensity mapping in the reciprocal space of the $(2,0,0)$ Bragg peak, a main reflection of the parent Pm3m cubic phase. Figure 3 -a shows the elastic intensity mapping at $\mathrm{T}=180 \mathrm{~K}$ well within the $\mathrm{I} 4 / \mathrm{mcm}$ tetragonal phase. One clearly sees a splitting of the Bragg peak reflection of the parent cubic phase below $T_{c}$. The splitting reveals the progressive build-up of at least 3 symmetry-related crystalline domains. This experimental signature is consistent with the coupling between the lattice strain and the phase transition order parameter.

Indeed, in oxide perovskites, $\mathrm{Pm}-3 \mathrm{~m}$ to Pnma and Pm-3m to I4/mcm instabilities are competing, in the same way as in $\mathrm{MAPbI}_{3}$ and $\mathrm{MAPbBr}_{3}$. The cubic $(\mathrm{Pm}-3 \mathrm{~m})$ to orthorhombic (Pnma) instability is related to the simultaneous condensation of $\mathrm{R}_{4}^{+}$and $\mathrm{M}_{3}{ }^{+}$rotational modes of the lead halide octahedra, whereas the cubic $(\mathrm{Pm}-3 \mathrm{~m})$ to tetragonal $(\mathrm{I} 4 / \mathrm{mcm})$ instability only involves an order parameter of the $\mathrm{R}_{4}^{+}$type. More, the $\mathrm{Pm}-3 \mathrm{~m}$ to $\mathrm{I} 4 / \mathrm{mcm}$ instability is continuous and occurs at high temperature (about $T_{c}=330 \mathrm{~K}$ for $\mathrm{MAPbI}_{3}$ and $\mathrm{T}_{\mathrm{c}}=225 \mathrm{~K}$ for $\mathrm{MAPbBr}_{3}$ ). A Pnma phase is only observed at low temperature in both $\mathrm{MAPbI}_{3}$ and $\mathrm{MAPbBr}_{3}$ crystals. From the symmetry analysis, a linearquadratic coupling is also predicted in the cubic phase between the linearly coupled $\mathrm{R}_{4}{ }^{+}$order parameters and two components of the strain tensor, $\varepsilon_{1}+\varepsilon_{2}+\varepsilon_{3}$ and $2 \varepsilon_{3}-\varepsilon_{1}-\varepsilon_{2}$ that correspond to the $\Gamma_{1}{ }^{+}$and $\Gamma_{3}{ }^{+}$IR, respectively. The cubic to tetragonal phase transition thus presents an improper ferroelastic character.

Next, we performed inelastic neutron scattering measurements around the $(2,0,0)$ main Bragg peak of the cubic phase. Preliminary results are shown at room temperature on figure 3-b. It is the first direct experimental evidence of longitudinal (LA) and transverse (TA) acoustic phonon dispersions in 3D hybrid perovskites. The longitudinal and transverse mode have been measured at $\mathrm{Q}=(2.05,0,0)$ and $\mathrm{Q}=(2,-0.1,-0.1)$ respectively. For both scans, a peak is clearly sizeable above a large quasi-elastic background related to the incoherent scattering of hydrogen. The two spectra are shown with fitting curves that integrate the phonon peak component with damped harmonic oscillator, the quasielastic peak component $(\mathrm{QE})$ and the background $(\mathrm{BG})$. The peak components are also convoluted with the instrumental resolution function (of the order of $0.2 \mathrm{meV}$ here). A strong damping is also observed for both phonon lines; however, it affects more dramatically the longitudinal mode. A detailed temperature investigation is necessary to understand the influence of strain, lead halide octaedra or MA molecular stochastic rotations on the damping of LA and TA acoustic phonons.

citation: Jacky Even ; Serge Paofai ; Philippe Bourges ; Antoine Létoublon ; Stéphane Cordier, Olivier Durand, Claudine Katan, " Carrier scattering processes and low energy phonon spectroscopy in hybrid perovskites crystals ", Proc. SPIE 9743, Physics, Simulation, and Photonic Engineering of Photovoltaic Devices V, 97430M (March 14, 2016); doi:10.1117/12.2213623; http://dx.doi.org/10.1117/12.2213623 


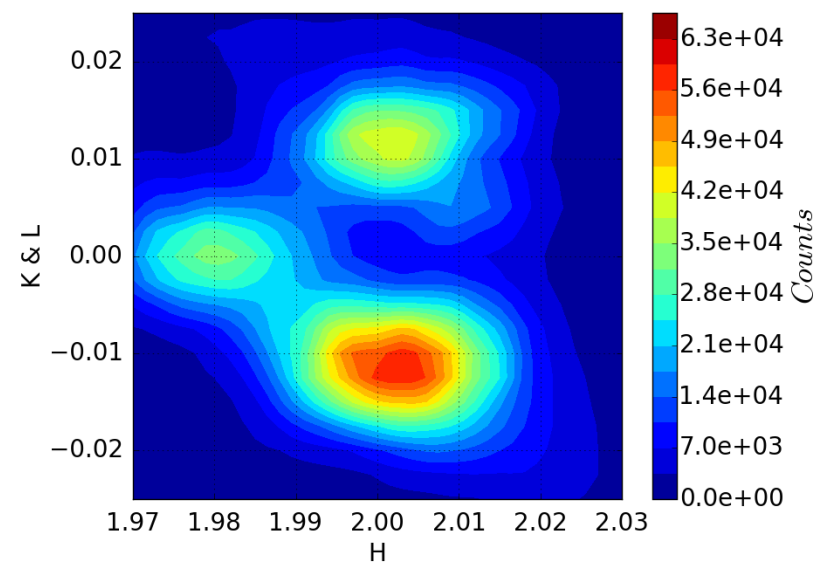

(a)

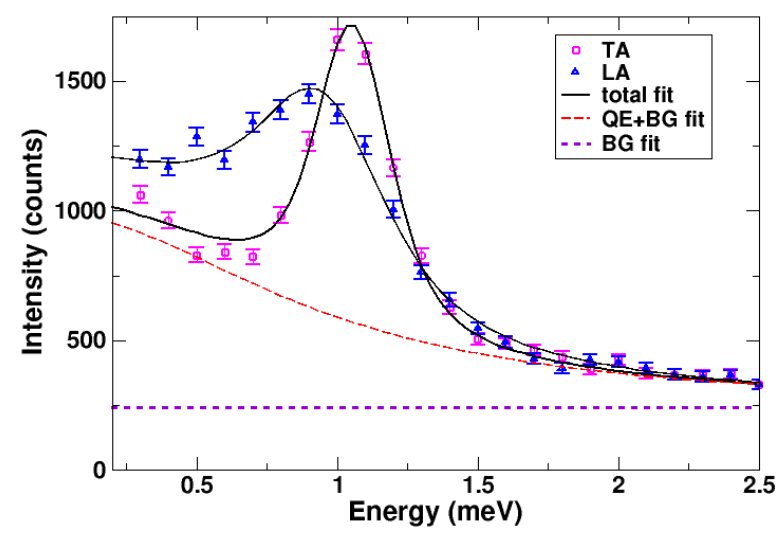

(b)

Figure 3: (a) Elastic intensity mapping of $\mathrm{MAPbBr}_{3}$ in the $\mathrm{I} 4 / \mathrm{mcm}$ tetragonal phase $(\mathrm{T}=180 \mathrm{~K})$, around the $(2,0,0) \mathrm{Bragg}$ peak of the parent $\mathrm{Pm} 3 \mathrm{~m}$ cubic phase. The mapping is performed in the $(\mathrm{H}, \mathrm{K}, \mathrm{K})$ ) scattering plane. (b) Room temperature inelastic neutron scattering spectra of $\mathrm{MAPbBr}_{3}$ measured close to the $(2,0,0)$ point of the reciprocal space. The longitudinal (LA) and transverse (TA) acoustic phonons are shown in the $\left(\mathbf{a}^{*},\left(\mathbf{b}^{*}+\mathbf{c}^{*}\right)\right)$ scattering plane. For both spectra, the total fitting curves are shown with background $(\mathrm{BG})$ and quasielastic $(\mathrm{QE})$ components.

\section{ULTRASONIC WAVES PROPAGATION IN MAPBBR}

We used a smaller $\left(\sim 0.2 \mathrm{~cm}^{3}\right)$ single crystal of $\mathrm{MAPbBr}_{3}$ synthetized, purified and grown according to Ref.[30], for an ultrasonic time-domain reflectometry experiment at room temperature using a longitudinal probe at $7.46 \mathrm{MHz}$ (figure 4). The single crystal was carefully chosen in order to yield a [001] flat surface with a crystal thickness equal to $3 \mathrm{~mm}$ (figure 4-b). A thin $(<0.1 \mathrm{~mm})$ gel layer was used between the probe and the crystal in order to ensure a maximum transmission and reflection of the ultrasonic pulse.

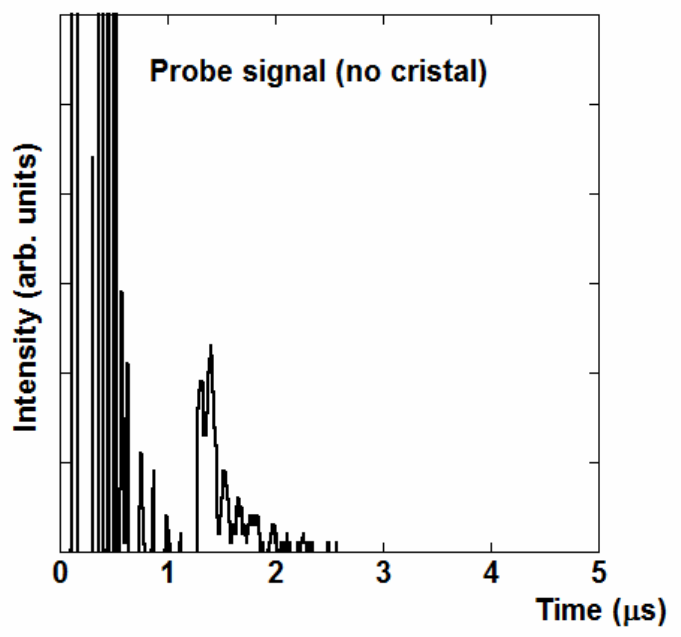

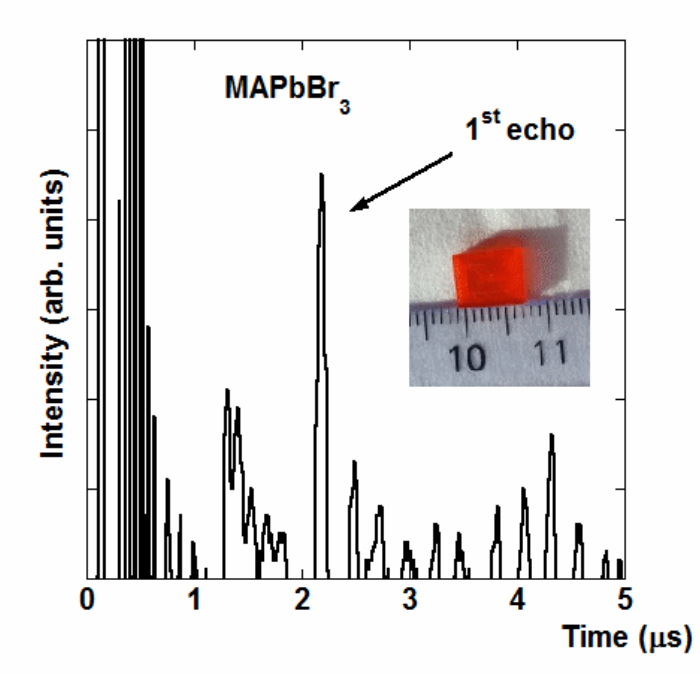

(b) (a) 
Figure 4: (a) Ultrasonic probe signal at $7.46 \mathrm{MHz}$ with full-wave rectification after the pulse emission at $\mathrm{t}=0$. (b) Ultrasonic reflected signal for a $\mathrm{MAPbBr}_{3}$ crystal (insert).

Figure 4-a shows the probe signal with full-wave rectification after the pulse emission at $\mathrm{t}=0$. Notice the parasitic signal in the $[1-2 \mu \mathrm{s}]$ time window. Figure 3-b shows the ultrasonic reflected signal for a $\mathrm{MAPbBr}_{3}$ crystal. Apart from the first echo, weaker parasitic replica are observed at higher times $(>2.5 \mu \mathrm{s})$. These fluctuating replica are related to the thin layer of gel. The longitudinal LA sound speed deduced from the ultrasonic measurements was found to be equal to $2900 \mathrm{~ms}^{-1} \pm 200 \mathrm{~ms}^{-1}$, in good agreement with measurements on the LA mode by neutron scattering (previous section).

\section{CONCLUSION}

A general symmetry-based framework has been introduced to understand the influence of various scattering processes on the charged carrier mobilities in 3D HOP such as $\mathrm{MAPbI}_{3}$ or $\mathrm{MAPbBr}_{3}$. The Fermi golden rule can be used to further evaluate numerically the influence of the symmetry-allowed scattering processes. Experimental data are lacking for that purpose about low-frequency acoustic and optical phonon modes, and molecular relaxational processes. Large single crystals of $\mathrm{MAPbBr}_{3}$ have been grown and used to perform neutron scattering and ultrasonic characterization of low frequency acoustic phonon in different energy ranges. Neutron scattering experiments further reveal that the $\mathrm{MAPbBr}_{3}$ crystal presents a coexistence of crystalline domains below the cubic to tetragonal phase transition at $T_{c}=225 \mathrm{~K}$. This observation is consistent with the improper ferroelastic character of the phase transition as predicted from the symmetry analysis of phonon and molecular relaxational modes.

\section{REFERENCES}

[1] Kojima, A., Teshima, K., Shirai, Y. \& Miyasaka, T., "Organometal Halide Perovskites as Visible-Light Sensitizers for Photovoltaic Cells," J. Am. Chem. Soc. 131 (17), 6050-6051 (2009).

[2] Im, J. H., Lee, C. R., Lee, J. W., Park, S. W. \& Park, N. G., “6.5\% Efficient Perovskite Quantum-Dot-Sensitized Solar Cell." Nanoscale, 3(10), 4088-4093 (2011).

[3] Lee, M. M., Teuscher, J., Miyasaka, T., Murakami, T. N. \& Snaith, H. J., "Efficient Hybrid Solar Cells Based on Meso-Superstructured Organometal Halide Perovskites," Science 338, 643-647 (2012).

[4] Kim, H. S., Lee, C. R., Im, J. H., Lee, K. B., Moehl, T., Marchioro, A., Moon, S. J., Humphry-Baker, R., Yum, J. H., Moser, J. E., Grätzel, M. \& Park, N-G., "Lead Iodide Perovskite Sensitized All-Solid-State Submicron Thin Film Mesoscopic Solar Cell with Efficiency Exceeding 9\%," Sci. Rep. 2, 591-591-7 (2012).

[5] Burschka, J., Pellet, N., Moon, S. J., Humphry-Baker, R., Gao, P., Nazeeruddin, M.K. \& Grätzel, M., "Sequential deposition as a route to high-performance perovskite-sensitized solar cells," Nature 499, 316-319 (2013).

[6] Liu, M., Johnston, M. B. \& Snaith, H. J., "Efficient planar heterojunction perovskite solar cells by vapour deposition," Nature 501, 395-398 (2013).

[7] http://www.nrel.gov/ncpv/images/efficiency_chart.jpg

[8] Mosconi, E., Amat, A., Nazeeruddin, Md. K., Grätzel, M., \& De Angelis, F., "First-principles modeling of mixed halide organometal perovskites for photovoltaic applications," J. Phys. Chem. C 117, 13902-13913 (2013).

[9] Even, J., Pedesseau, L., Jancu, J.-M. \& Katan, C., "Importance of spin-orbit coupling in hybrid organic/inorganic perovskites for photovoltaic applications," J. Phys. Chem. Lett. 4, 2999-3005 (2013).

[10] Brivio, F., Walker, A. B., \& Walsh, A, "Structural and electronic properties of hybrid perovskites for highefficiency thin-film photovoltaics from first-principles," Appl. Phys. Lett. Mat. 1, 042111-1-042111-5 (2013).

[11] Giorgi, V., Fujisawa, J.-I., Segawa, H., \& Yamashita, K., "Small photocarrier effective masses featuring ambipolar transport in methylammonium lead iodide perovskite: A density functional analysis," J. Phys. Chem. Lett. 4, 4213$4216(2013)$.

[12] Poglitsch, A. \& Weber, D. J., "Dynamic disorder in methylammoniumtrihalogenoplumbates (II) observed by millimeter-wave spectroscopy.” J. Chem. Phys. 87, 6373-6378 (1987).

citation: Jacky Even ; Serge Paofai ; Philippe Bourges ; Antoine Létoublon ; Stéphane Cordier, Olivier Durand, Claudine Katan, " Carrier scattering processes and low energy phonon spectroscopy in hybrid perovskites crystals ", Proc. SPIE 9743, Physics, Simulation, and Photonic Engineering of Photovoltaic Devices V, 97430M (March 14, 2016); doi:10.1117/12.2213623; http://dx.doi.org/10.1117/12.2213623 
[13] Onoda-Yamamuro, N., Matsuo, T. \& Suga, H. "Calorimetric and IR Spectroscopic Studies of Phase-Transitions in Methylammonium Trihalogenoplumbates-(II).” J. Phys Chem. Solids 51, 1383-1392 (1990).

[14] Onoda-Yamamuro, N., Matsuo, T. \& Suga, H. "Dielectric study of CH3NH3PbX3 (X=Cl, Br, I)." J. Phys. Chem. Solids 53, 935-939 (1992).

[15] Wasylishen, R. E., Knop, O. \& Macdonald, J. B. "Cation rotation in methylammonium lead halides." Solid State Comm. 56, 581-582 (1985).

[16] Even, J., Pedesseau, L. \& Katan, C., "Analysis of multivalley and multibandgap absorption and enhancement of free carriers related to exciton screening in hybrid perovskites," J. Phys. Chem. C 118, 11566-11572 (2014).

[17]Fang, H.-H., Raissa, R., Abdu-Aguye, M., Adjokatse, S., Blake, G.R., Even, J. \& Loi, M.A. "Photophysics of Organic-Inorganic Hybrid Lead Iodide Perovskite Single Crystals,” Adv. Func. Mat., 25, 2378-2385 (2015).

[18] Miyata, A., Mitioglu, A., Plochocka, P., Portugall, O., Wang, J. T. S. , Stranks, S. D., Snaith, H. J. \& Nicholas, R. J. "Direct Measurement of the Exciton Binding Energy and Effective Masses for Charge carriers in an OrganicInorganic Tri-halide Perovskite," Nature Physics, 11, 582-587 (2015).

[19] Even, J., "Pedestrian Guide to Symmetry Properties of the Reference Cubic Structure of 3D All-inorganic and Hybrid Perovskites," J. Phys. Chem. Lett. 6, 2238-2242 (2015).

[20] Even, J.; Carignano, M. \& Katan, C. "Molecular disorder and translation/rotation coupling in the plastic crystal phase of hybrid perovskites." Nanoscale, DOI: 10.1039/C5NR06386H, (2016).

[21] Lynden Bell, R.M. \& Michel, K.H. "Translation-rotation coupling, phase transitions, and elastic phenomena in orientationally disordered crystals." Rev. Mod. Phys., 721-762 (1994).

[22] Niemann, R.G., Kontos, A. G., Palles, D., Kamitsos, E.I., Kaltzoglou, A., Brivio, F., Falaras, P. \& Cameron, P.J. "Halogen Effects on Ordering and Bonding of $\mathrm{CH} 3 \mathrm{NH} 3+$ in $\mathrm{CH} 3 \mathrm{NH} 3 \mathrm{PbX} 3(\mathrm{X}=\mathrm{Cl}, \mathrm{Br}, \mathrm{I})$ Hybrid Perovskites: A vibrational spectroscopic study", J. Phys. Chem. C, DOI: 10.1021/acs.jpcc.5b11256 (2016).

[23] Fujii, Y., Hoshino, S., Yamada, Y. \& Shirane, G. "Neutron-scattering study on phase transitions of CsPbCl3." Phys. Rev. B 9, 4549-4559 (1974).

[24] Sinha, M.M. \& Wakamura, K. "Study of phonons and normal mode analysis of perovskite-type superionic conductors." Solid State Ionics 136, 1345-1350 (2000).

[25] Baikie, T., Barrow, N. S., Fang, Y., Keenan, P. J., Slater, P.R., Piltz, R.O., Gutmann, M. S., Mhaisalkar, G. \& White, T.J. "A combined single crystal neutron/X-ray diffraction and solid-state nuclear magnetic resonance study of the hybrid perovskites CH3NH3PbX3 (X = I, Br and Cl).” J. Mater. Chem. A 3, 9298-9307 (2015).

[26] Weller, M. T., Weber, O. J., Henry, P.F., Di Pumpo, A.M. \& Hansen, T.C. "Complete structure and cation orientation in the perovskite photovoltaic methylammonium lead iodide between 100 and $352 \mathrm{~K}$." Chem. Commun. 51, 4180-4183 (2015).

[27] Leguy, A. M. A., Frost, J.M., McMahon, A.P., Sakai, V.G., Kochelmann, W., Law, C.H., Li, X., Foglia, F., Walsh,A., O'Regan, B.C., Nelson, J., Cabral, J.T. \& Barnes, P.R.F. "The dynamics of methylammonium ions in hybrid organic-inorganic perovskite solar cells." Nat. Commun., 6, 7124 (2015).

[28] Chen, T., Foley, B.J., Ipek, B., Tyagi, M., Copley, J. R. D., Brown, C.M., Choi, J.J. \& Lee, S. "Rotational dynamics of organic cations in the CH3NH3PbI3 perovskite." Phys. Chem. Chem. Phys., 17, 31278-31286 (2015).

[29] Swainson, I.P., Stock, C., Parker, S.F., Van Eijck, L., Russina, M. \& Taylor, J.W. "From soft harmonic phonons to fast relaxational dynamics in CH3NH3PbBr3.” Phys. Rev. B, 92, 100303(R) (2015).

[30] Saidaminov, M. I., Abdelhady, A. L., Murali, B., Alarousu, E., Burlakov, V. M., Peng, W., Dursun, I., Wang, L., He, Y., Maculan, G., Goriely, A., Wu, T., Mohammed, O. F. \& Bakr, O.M. "High-quality bulk hybrid perovskite single crystals within minutes by inverse temperature crystallization," Nature Comm. 6, 7586 (2015).

[31] Toudic, B., Lefort, R., Ecolivet, C., Guérin, L., Currat, R., Bourges, P. \& Breczewski, T. "Mixed Acoustic Phonons and Phase Modes in an Aperiodic Composite Crystal.” Phys. Rev. Lett. 107, 205502 (2011).

[32] Swainson, I.P., Hammond, R.P., Soullière, C., Knop, O. \& Massa, W. "Phase transitions in the perovskite methylammonium lead bromide, CH3ND3PbBr3.” J. Solid State Chem. 176, 97 (2003).

citation: Jacky Even ; Serge Paofai ; Philippe Bourges ; Antoine Létoublon ; Stéphane Cordier, Olivier Durand, Claudine Katan, " Carrier scattering processes and low energy phonon spectroscopy in hybrid perovskites crystals ", Proc. SPIE 9743, Physics, Simulation, and Photonic Engineering of Photovoltaic Devices V, 97430M (March 14, 2016); doi:10.1117/12.2213623; http://dx.doi.org/10.1117/12.2213623 